\title{
Unusual localizations of human hydatid disease in Hamedan province, west of Iran
}

\author{
N. A. AHMADI ${ }^{1}$, M. HAMIDI ${ }^{2}$ \\ ${ }^{1}$ Department of Medical Lab Technology, and Proteomics Research Center, Shahid Beheshti University of Medical \\ Sciences, Tehran, Iran, E-mail: nayebalia@yahoo.com,nayebalia@sbmu.ac.ir; ${ }^{2}$ Biology Deptartment, Shahed \\ University, Khalij Fars Express way, Tehran, Iran
}

\section{Summary}

Cystic echinococcosis (CE) is endemic in many parts of Iran. It may develop in almost any part of the human body, though the location is mostly hepatic and followed by pulmonary. This study was carried out on the 24 patients diagnosed with unusual localizations of hydatidosis among the patients admitted to the university hospitals in Hamedan province, west of Iran, during 14-year period. The cases were aged $6-73$ years, the majority being aged $40-59$ years $(33.3 \%)$. Ten of the patients diagnosed with unusually located hydatid disease were men, fourteen were women. The over-representation of housewives $(50.0 \%)$ and followed by farmers $(20.8 \%)$ among the cases was significant. Similarly, rural dwellers were also over-represented among the cases (54.2\% vs. $45.8 \%$ ).

Most (19 case) of the cases had been diagnosed just by ultrasonography, but 3 case had only been diagnosed by computed tomographic (CT) scans, and another 2 had had both ultrasonography and CT scans. Spleen and kidneys, followed by abdominal/peritoneal cavity, were the organs where hydatid disease was most frequently observed after the liver and lung. The splenic cysts were solitary in 4 cases, associated with a hepatic cyst in 1 and with pelvic and abdominal wall cysts in 1 . The renal cysts were usually isolated without any other organ involvement, and there was only one case of associated with hydatid disease in liver. In addition, findings in brain, mesenterium, breast, choledochus, bladder wall and inguinal area are also discussed.

These results confirm that $\mathrm{CE}$ should be considered in the differential diagnosis of any cystic mass in all anatomic locations (including unusual localizations), especially when occurring in areas where the disease is endemic.

Keywords: hydatid disease; Echinococcus granulosus; unusual localizations; Iran

\section{Introduction}

Cystic echinococcosis (CE), caused by the metacestode of Echinococcus granulosus, is recognized as one of the world's major zoonoses (Thompson, 2001; Guzel et al., 2008; Ahmadi \& Hamidi, 2008). CE has been reported from all countries in the Middle East (including Iran) and Arabic North Africa from Morocco to Egypt (Sadjjadi, 2006; Ahmadi \& Hamidi, 2008). Hydatid cyst is characterized by cystic lesions with clear boundaries, which can be observed in all parts of the body. The hydatid cyst follows a silent clinical course until the cyst grows larger and gives pressure symptoms. Differential diagnosis between human $\mathrm{CE}$ and alveolar echinococcosis (AE) has significant implications for epidemiologic studies and treatment of these diseases, especially in the regions where both species occur sympatrically. Imaging methods, including ultrasonography, computerized tomography (CT), and magnetic resonance imaging (MRI), can provide a speciesspecific differential diagnosis in many cases, but diagnosis may be problematic in others (Ito et al., 1999; Çöl et al., 2003). Serodiagnostic tests based on antibody detection exhibit high sensitivity and reasonable specificity, for example detection of antibodies to the specific Em18 antigen, either immunoblot or ELISA, appears to be the most specific for AE. Diagnosis in areas where both AE and CE are endemic, should be carried out as a combination of imaging methods and serologic tests (Ito et al., 1999; Ito et al., 2003). Glycoproteins (GPs) purified from cyst fluid of Taenia solium are highly specific for diagnosis of $T$. solium neuorcysticercosis (NCC), and specific serological test for differentiation of NCC from echinococcosis is GPELISA or GP-immunoblot (Ito et al., 2003).

The most common organ affected by hydatid disease is the liver $(55-70 \%)$ followed by the lungs $(20-35 \%)$ and the two organs are affected simultaneously in about $5-13 \%$ of cases (Abu-Eshy, 1998; Ahmadi \& Hamidi, 2008). The kidneys, spleen, abdominal/peritoneal cavity, mesentery, 
Table 1. Unusual localizations of hydatid cysts in confirmed human cystic echinococcosis cases from Hamedan province, Iran.

\begin{tabular}{lccc}
\hline Location & $\begin{array}{c}\text { Number } \\
\text { of cases }\end{array}$ & $\begin{array}{c}\text { \% of cases with } \\
\text { unusual site }\end{array}$ & $\begin{array}{c}\text { \% of all patients with } \\
\text { hydatidosis }(\mathrm{N}=179)\end{array}$ \\
\hline Spleen $^{\mathrm{a}}$ & 5 & 20.83 & 2.79 \\
Kidney $^{\mathrm{a}}$ & 5 & 20.83 & 2.79 \\
Abdominal/peritoneal cavity $^{\mathrm{a}}$ & 4 & 16.67 & 2.23 \\
Gallbladder $^{\mathrm{a}}$ & 2 & 8.33 & 1.11 \\
(Spleen+pelvis+abdominal wall) $^{\mathrm{b}}$ & 1 & 4.17 & 0.56 \\
Pancreas $_{\text {Brain }}$ & 1 & 4.17 & 0.56 \\
Breast & 1 & 4.17 & 0.56 \\
Bladder wall & 1 & 4.17 & 0.56 \\
Mesenterium & 1 & 4.17 & 0.56 \\
Inguinal canal & 1 & 4.17 & 0.56 \\
Choledochus & 1 & 4.17 & 0.56 \\
\hline Total & 1 & 4.17 & 0.56 \\
\hline
\end{tabular}

${ }^{\mathrm{a} I n}$ two cases plus liver cyst.

${ }^{\mathrm{b}}$ This case was together with liver cyst.

pancreas, brain, breast, and bladder wall are less frequent sites (Amr et al., 1994; Lucandri et al., 1994; Prousalidis et al., 1998; Kireşi et al., 2003; Çöl et al., 2003).

In Iran, the adult stage of infection is commonly found among dogs, wolves, foxes and jackals (Fallah et al., 1995; Eslami \& Hosseini, 1998; Dalimi et al., 2002), and the larval stage among livestock (Arbabi et al., 1998; Ansari-Lari, 2005; Dalimi et al., 2002; Ahmadi, 2005). There are several reports of human infection with the parasite from different provinces in Iran (Sadrieh et al., 1967; Ahmadi \& Dalimi 2006; Baharsefat et al., 2007; Pezeshki et al., 2007; Ahmadi \& Hamidi, 2008). Hamedan province includes pastures and agricultural lands that support population of farm animals. In Hamedan province, the very high prevalence of E. granulosus was reported from stray dogs (Fallah et al., 1995), and also high infection rate of hydatid cyst was observed in slaughtered livestock (Arbabi et al., 1998).

In this study, we investigated on patients treated with unusual localizations of hydatidosis among the patients admitted to the university hospitals in Hamedan province, west of Iran, during $1992-2006$.

Table 2. Age and sex distribution of unusual localizations of human cystic echinococcosis in Hamedan province, Iran.

\begin{tabular}{|c|c|c|c|c|c|c|}
\hline \multirow[t]{2}{*}{ Age } & \multicolumn{2}{|c|}{ Male } & \multicolumn{2}{|c|}{ Female } & \multicolumn{2}{|c|}{ Both } \\
\hline & $\mathrm{N}$ & $(\%)$ & $\mathrm{N}$ & $(\%)$ & $\mathrm{N}$ & $(\%)$ \\
\hline$<20$ & 3 & $(12.5)$ & 2 & $(8.3)$ & 5 & $(20.8)$ \\
\hline $20-39$ & 3 & $(12.5)$ & 4 & (16.7) & 7 & $(29.2)$ \\
\hline $40-59$ & 3 & (12.5) & 5 & (20.8) & 8 & $(33.3)$ \\
\hline$>=60$ & 1 & $(4.2)$ & 3 & $(12.5)$ & 4 & (16.7) \\
\hline Total & 10 & $(41.7)$ & 14 & $(58.3)$ & 24 & $(100.0)$ \\
\hline
\end{tabular}

$\mathrm{N}$ : number of cases

\section{Patients and Methods}

A retrospective, descriptive study was carried out on 24 patients diagnosed with unusual localizations of hydatidosis among 179 patients with human CE who admitted to the university hospitals in Hamedan province, an endemic region of Iran, during 1992 - 2006. These university hospitals are teaching hospitals under the Hamedan University of Medical Sciences and admit patients from different cities of province.

The patients were evaluated regarding their age, sex, job, site of cyst, residency (rural/urban). Radiological data, including ultrasonography (USG) and computed tomography (CT) scan records, were collected from the files.

\section{Results}

Among 179 patients who had hydatid cyst operations in Hamedan university hospitals, 24 (13.4 \%) had a localization other than in the liver and lungs. The organ distribution of the unusually located hydatid cysts is shown in Table 1. Spleen and kidneys, followed by abdominal/peritoneal cavity, are the organs where hydatid disease is most frequently observed after the liver and lung. Eighteen cases $(75 \%)$ had a single organ of infection, and 6 patients showed multiple organs involvement.

The majority of the patients diagnosed with unusually located hydatid disease were female $(58.3 \%)$, and remainder of cases were male. Age and sex distribution are shown in Table 2. The age of the cases ranged from 6 to 73 years. Age groups 40 - 59 were the most affected. Housewives had the highest rate of infection (12 cases, $50 \%$ ) followed by farmers ( 5 cases, $20.8 \%$ ), workers ( 2 cases), school students ( 2 cases), government employees ( 2 cases) and free job ( 1 case). As to residency, thirteen patients (54.2\%) came from rural areas, while eleven cases $(45.8 \%)$ were from urban areas. 
In Hamedan province, the patients were evaluated using USG followed by CT imaging only. Diagnosis by USG, CT-scan alone and in conjunction with both methods constituted $19(79.2 \%), 3(12.5 \%)$ and $2(8.3 \%)$ cases of the unusually located hydatid cysts, respectively.

\section{Discussion}

Hydatid disease is still a problem in endemic areas of the world including Iran. Studies in Hamedan province in western Iran indicated that $49 \%$ of the stray dogs were infected with E. granulosus (Fallah et al., 1995), and also $27.5 \%$ of the sheep, $16.1 \%$ of the goats, and $25.9 \%$ of the cattle were infected with hydatid cyst (Arbabi et al., 1998). Hamedan province in west of Iran, has the potential of high prevalence of this disease due to existence of animal husbandries and farmer jobs, and also presence of numerous dogs including stray dogs. To date, no study has been performed on unusual localizations of hydatidosis in the region.

The disease usually involves the liver and lungs, but any organ or tissue can potentially be involved by hydatid disease. There are no specific local or general symptoms and signs of hydatid disease (Çöl et al., 2003). The majority of infestations are diagnosed following incidental findings at radiographic examination for unrelated complaints. Direct radiography, eosinophilia, and the immunoserologic tests may be useful for diagnosis, but abdominal USG and CT-scans are most useful for establishing the diagnosis of hydatid disease (Çöl et al., 2003). In Hamedan province the favourable and available imaging method was USG followed by the CT-scan. CT scan was done for 3 cases and all of them showed positive results for hydatid cysts. Diagnosis by USG alone and in conjunction with CT-scan constituted $19(79.2 \%)$ and 2 cases $(8.3 \%)$ of the total unusual cases, respectively. Therefore, in countries where hydatid disease is endemic, clinicians and radiologists often come across this disease and, they take it into consideration in the differential diagnosis.

Apart from the liver and lung, the localizations where hydatid disease is considerably seen are the kidneys, spleen, abdominal/peritoneal cavity, gallbladder, breast and brain (Çöl et al., 2003; Amr et al., 1994; Kireşi et al., 2003; Sadrieh et al, 1967; Balik et al., 2001). In our series of patients, spleen and kidney localizations, equal in number, were predominant among the unusually located hydatid cysts. Such results were reported in Turkey (Col et al., 2003). The splenic involvement by CE is rare and accounts for about $2 \%$ of all patients (Çöl et al., 2003; Amr et al., 1994; Kireşi et al., 2003). In the present study, the splenic cysts were solitary in 4 cases, associated with a hepatic cyst in 1 and with pelvic and abdominal wall cysts in 1 . In this study, hydatid disease of the spleen represented $2.8 \%$ of all hydatidosis cases. This value is similar to those reported in Iran (Pezeshki et al, 2007; Sadrieh et al., 1967), Jordan (Amr et al., 1994) and Turkey (Çöl et al., 2003).

Urinary tract involvement is not common, corresponding to only $2-4 \%$ of all patients with CE (Feki et al., 2008). Renal hydatidosis is usually single and included $2-3 \%$ of all cases (Kireşi et al., 2003). In the present study, hydatid cysts of the kidneys were mainly isolated without any other organ involvement, but there was one case of associated with hydatid disease in liver. The kidney was affected in $2.8 \%$ of all the patients with CE. This is close to the rate reported from Jordan by Amr et al. (1994), who reported 10 cases of renal hydatid deases $(3.5 \%)$ out of a total of 306. Among the unusual presentations of the echinococcal cyst, the occurrence of bladder hydatid cysts is exceptional with only a few case reports found in the literature (Feki et al., 2008; Sallami et al., 2005; Cherkaoui et al., 2002; Deodhar et al., 1986; Fuloria et al., 1975). It is observed approximately in $0.2-0.5 \%$ of cases (Amr et al., 1994, Feki et al., 2008). In our series, hydatid disease of bladder wall showed 1 patient of all cases.

Hydatid cysts of the peritoneal cavity are usually accompanied by liver hydatid disease. In this survey, two cases of hydatid cyst of the abdominal/peritoneal cavity were solitary, and two patients in this subgroup presented multiple cysts as concomitant liver hydatidosis. In our study, only four patients $(2.2 \%)$ out of total cases had abdominal/peritoneal cysts. This is close to the rate reported from Turkey (Balik et al., 2001), Jordan (Amr et al., 1994) and Iran (Sadrieh et al., 1967).

Cerebral hydatid disease represents only $2 \%$ of all cerebral space-occupying lesions even in the countries where the disease is endemic (Kireşi et al., 2003). Hydatid cysts of the brain are usually accompanied by liver and/or lung hydatid disease, while in this work, the brain cyst was solitary and primary in one case.

Hydatid disease of the breast is rare, accounting for only $0.27 \%$ of all patients with CE (Farrokh et al., 2007; Vega et al., 1994). The breast can be the only primary site or part of disseminated hydatidosis. During the last ten years, six patients with primary hydatid cysts of the breast were reported from Iran in literature (Farrokh et al., 2007). Herein, we have reported one another case with this rare condition in Hamedan, west of Iran.

Hydatid cyst of the inguinal area, resembling a hernia is a rarity presenting at an unusual site. We observed one case primary hydatid cyst of the inguinal canal. In a previous survey, inguinal hydatid disease was reported from Iran by Kouhsari and Manzar (1998).

Primary CE of the pancreas is extremely rare $(0.2-2 \%)$ and mainly solitary (Kireşi et al., 2003). In our series, primary hydatid disease of the pancreas represents 1 case $(0.6 \%)$ of all hydatidosis cases as solitary. This result is similar to those reported in Iran (Pezeshki et al., 2007; Sadrieh et al., 1967) and Turkey (Balik et al., 2001).

Hydatid disease of the galbllader is rare, and we found two cases. The gallbladder cysts were solitary and primary in 1 case, associated with a hepatic cyst in 1. Sadrieh et al. (1967) reviewed 150 cases of hydatid diseases in Iran, of which only three cases were galbllader hydatid disease. Primary mesentery hydatidosis is extremely rare. In this study observed only one primary mesenteric cyst. In com- 
parison, 3 of 306 cases in Jordan (Amr et al., 1994), one of 389 cases in Turkey (Balik et al., 2001) located in the mesentery.

Among the unusual presentations of the echinococcal cyst, the occurrence of primary choledochal hydatid cyst is exceedingly rare with only a few case reports found in the literature (Gupta, 1989; Alladi et al., 1995; Otgun et al., 2003). Herein, we report on the first case of primary choledochal hydatid cyst in Iran.

In the present study, the age of the cases ranged from 6 to 73 years, and the more affected age groups were $40-59$ and then $20-39$ years (Table 2). These results are similar to a report of Iran (Baharsefat et al, 2007; Pezeshki et al, 2007). However, Amr et al. (1994) in Jordan found more hydatid surgeries in the age group of 16-30 year old.

Overall incidence of CE was considerably higher in female patients (see Table 2). These results are consistent with reports from Iran (Baharsefat et al, 2007; Pezeshki et al, 2007), Jordan (Amr et al., 1994) and Iraq (Saeed et al., 2000).

In studied area, most of the suffering individuals from $\mathrm{CE}$ were housewives $(50.0 \%)$, and is similar to those reported in Iran (Baharsefat et al, 2007; Pezeshki et al, 2007), Jordan (Amr et al., 1994) and Iraq (Saeed et al., 2000). The prevalence of CE surgery in the rural areas (54.2\%) was higher than the urban areas (45.8). Such as results, were reported in previous studies (Amr et al., 1994; Baharsefat et al., 2007; Pezeshki et al., 2007; Saeed et al., 2000).

In conclusion, unusual localizations $\mathrm{CE}$ should be considered in the differential diagnosis of any cystic mass in all anatomic locations, especially when occurring in areas where the disease is endemic. Also, CE remains an important health problem in many areas of Iran, including Hamedan province (west of Iran). Although there is clearly a need for a careful and nation-wide survey of CE in Iran, existing hospital records can help determine the unusual localizations of hydatid disease on a regional scale and thus help target control and prevention activities.

\section{Acknowledgements}

Hereby the authors wish to express their appreciation to all colleagues cooperating in conducting this study including staff of the university hospitals in Hamedan.

\section{References}

ABU-Eshy, S. A. (1998): Some rare presentations of hydatid cyst (Echinococcus granulosus). J. R. Coll. Surg. Edinb., 43: 347 - 352

AHMADI, N. A. (2005): Hydatidosis in camels (Camelus dromedarius) and their potential role in the epidemiology of Echinococcus granulosus in Iran. J. Helminthol., 79: $119-125$

AhMADI, N., DALimi, A. (2006): Characterization of Echinococcus granulosus isolates from human, sheep and camel in Iran. Inf. Gen. Evol., 6: 85 - 90

Ahmadi, N. A., HAMIDI, M. (2008): A retrospective analy- sis of human cystic echinococcosis in Hamedan province, an endemic region of Iran. Ann. Trop. Med. Parasitol., $102,603-609$

Alladi, A., SAOJI, R., Ramachandra, C., D'CruZ, A. J. (1995): Hydatid cyst: another differential diagnosis of choledochal cyst. Pediatr. Surg. Int., 10: $422-423$

Amr, S. S., Amr, Z. S., JitAwI, S., AnNAB, H. (1994): Hydatidosis in Jordan: an epidemiological study of 306 cases. Ann. Trop. Med. Parasitol., 88: 623 - 627

ANSARI-LARI, M. (2005): A retrospective survey of hydatidosis in livestock in Shiraz, Iran, based on abattoir data during 1999-2004. Vet. Parasitol., 133: 119 - 123

Arbabi, M., Massoud, J., Dalimi-Asl, A., SAdJJAdI, S. M. (1998): Prevalence of hydatidosis in slaughtered animals in Hamedan. Daneshvar Sci. Res. J. Shahed Univ., 5: $57-61$

Baharsefat, M., Massoud, J., Mobedi, I., FarahnaK, A., RoKNI, M. B. (2007): Seroepidemiology of human hydatidosis in Golestan province, Iran. Iranian J. Parasitol., 2: $20-24$

Balik, A. A., Celebi, F., Basoglu, M., Oren, D., Yildirgan, I., ATAMANALP, S. S. (2001): Intra-abdominal extrahepatic echinococcosis. Surg. Today, 31: $881-884$ CherkaOui, M. M., NASSAR, I., JRoundi, L., DAFIRI, R., IMANI, F. (2002): Hydatid disease of the urinary bladder: a case report. J. Radiol., 83: 45 - 46

ÇÖL, C., ÇÖL, M., LAFÇI, H. (2003). Unusual localizations of hydatid disease. Acta Med. Austriaca, 30: $61-64$

Dalimi, A., Motamedi, GH., HosseinI, M., MOHAMmadian, B., MAlaKi, H., GHAMARI, Z., GHAFFARI-FAR, F. (2002): Echinococcosis/hydatidosis in western Iran. Vet. Parasitol., 105: 161 - 171

Deodhar, S. D., Patel, V. C., Kirloskar, M. S. (1986): Hydatid disease of urinary bladder (a case report). J. Postgrad. Med., 32: 46B - 48B

ESLAMI, A., HosseINI, S. H. (1998): Echinococcus granulosus infection of farm dogs of Iran. Parasitol. Res., 84: $205-207$

FAllah, M., TAherkhani, H., SAdJjadi, S. M. (1995): Echinococcosis in stray dogs in Hamedan, west of Iran. Iranian J. Med. Sci., 29: 170 - 172

FARROKH, D., HASHEMI, J. B., ZANDI, B. (2007): Primary hydatid cyst of the breast: a case report. Iranian J. Radiol., 4: $159-162$

FeKi, W., Ghozzi, S., Khiari, R., Ghorbel, J., Elarbi, H., KHOUNI, H., RAIS, N. B. (2008): Multiple unusual locations of hydatid cysts including bladder, psoas muscle and liver. Parasitol. Int., 57: $83-86$

Fuloria, H. K., Jaiswal, M. S., Singh, R. V. (1975): Primary hydatid cyst of bladder. Br. J. Urol., 47: 192

GuptA, N. M. (1989): Primary choledochal echinococcosis. Aust. N. Z. J. Surg., 59: 668-670

Guzel, M., Yaman, M., Koltas, I. S., DemirkaziK, M., AKTAS, H. (2008) Detection of Echinococcus granulosus coproantigens in dogs from Antakya Province, Turkey. Helminthologia, 45:150 - 153

KIreşi, D. A., KARABACAKOĞLU, A., ÖDEV, K., KARAKÖSE, S. (2003): Uncommon locations of hydatid 
cysts. Acta Radiol., 44: $622-636$

KOUHSARI, M. R., MANZAR, H. A. (1998): Inguinal Hydatid cyst: report of a rare case. Med. J. Islam. Rep. Iran, 12: 177 - 179

Lucandri, G., D’Elia, G., Chiavellati, L., Sterpetti, A., Meloni, E., Canuti, W., Balducci, G., Ziparo, V., STIPA, F, STIPA, S. (1994): Unusual location of hydatid cysts: clinical and therapeutic aspects. G. Chir., 15: $529-$ 537

Otgun, I., Karnak, I., Haliloglu, M., SenocaK, M. E. (2003): Obstructive jaundice caused by primary choledochal hydatid cyst mimicking radiologically choledochal cyst. J. Pediatr. Surg., 38: 256 - 258

Pezeshio, A., Kia, E. B., Gholizadeh, A., Koohzare, A. (2007): An analysis of hydatid cyst surgeries in Tehran Milad hospital, Iran, during 2001-2004. Pak. J. Med. Sci., 23: $138-140$

Prousalidis, J., Tzardinoglou, K., Sgouradis, L., Katsohis, C., Aletras, H. (1998): Uncommon sites of hydatid disease. World J. Surg., 22: $17-22$

SADJJADI, S. M. (2006): Present situation of echinococcosis in the Middle East and Arabic North Africa. Parasitol. Int., 55: S197 - S202
SAdrieH, M., Dutz, W., NAVABPoOR, M. S. (1967): Review of 150 cases of hydatid cyst of the lung. Chest, 52: $662-666$

Saeed, I., Kapel, C., Saida, L. A., Willingham, L., NANSEN, P. (2000): Epidemiology of Echinococcus granulosus in Arbil province, northern Iraq, 1990-1998. J. Helminthol., 74: 83 - 88

Sallami, S., Nouira, Y., Kallel, Y., Gargouri, M., HORCHANI, A. (2005): Intravesical hydatid cyst. Urology, 66: 1110.e7-1110.e8

Vega, A., Ortega, E., Cavada, A., Garijo, F. (1994): Hydatid cyst of the breast: mammographic findings. $A$. $J$. R., 162: $825-826$

Ito, A., MA, L., Schantz, P. M., GotTstein, B., LiU, Y.H., Chai, J.-J., AbDel-Hafez, S. A., Altintas, N., Joshi, D. D., Lightowlers, M. W., Pawlowski, Z. S. (1999): Differential serodiagnosis for cystic and alveolar echinococcosis using fractions of Echinococcus granulosus cyst fluid (antigen B) and E. multilocularis protoscolex (Em18). Am. J. Trop.Med. Hy., 60: $188-192$

Ito, A., SAKo, Y., YAmasaki, H., Mamuti, W., NAKaya, K., NAKaO, M., IshiKaWA, Y. (2003): Development of Em18-immunoblot and Em18-ELISA for specific diagnosis of alveolar echinococcosis. Acta Tropica, 85: $173-182$ 